\title{
Níveis Plasmáticos Elevados de Lipoproteína(a) Correlacionados com a Gravidade da Doença Arterial Coronariana em Pacientes Submetidos à Angiografia
}

\author{
Increased Serum Levels of Lipoprotein(a) Correlated with the Severity of \\ Coronary Artery Disease in Patients Submitted to Angiography
}

Luciana Moreira Lima, Maria das Graças Carvalho, Andréia Assis Loures-Vale, Ana Paula Fernandes, Ana Paula Lucas Mota, Cirilo Pereira da Fonseca Neto, José Carlos Faria Garcia, Jamil Abdala Saad, Marinez de Oliveira Souza Universidade Federal de Minas Gerais e Hospital SOCOR de Belo Horizonte - Belo Horizonte, MG

\section{Objetivo}

Determinar os níveis plasmáticos de lipoproteína(a) e perfil lipídico de um grupo de indivíduos submetidos à angiografia coronariana, buscando estabelecer a possível correlação entre estes parâmetros e a gravidade da doença coronariana.

\section{MÉtodos}

Níveis plasmáticos de colesterol total, $\mathrm{HDL}_{\mathrm{C}}, \mathrm{LDL}_{\mathrm{C}}$, triglicérides, lipoproteína(a), apolipoproteínas A-I e B foram medidos em amostras de sangue de 17 indivíduos com ausência de ateromatose nas coronárias (controles), 12 indivíduos apresentando ateromatose leve/moderada e 28 indivíduos apresentando ateromatose grave.

\section{Resultados}

Não foram encontradas diferenças estatisticamente significativas entre as médias dos três grupos para os parâmetros avaliados, exceto para os níveis plasmáticos de lipoproteína(a) que apresentaram diferenças significativas entre as médias dos grupos controle, ateromatose leve/ moderada e ateromatose grave $(p<0,001)$.

\section{Conclusão}

As médias obtidas nos três grupos para Lp(a) sinalizam um aumento progressivo nos níveis plasmáticos deste parâmetro, de acordo com a gravidade da ateromatose coronariana. Estes achados sugerem a necessidade de estudos adicionais, visando obter suficiente evidência para a introdução rotineira da avaliação dos níveis de Lp(a) em laboratórios clínicos, no monitoramento de pacientes apresentando risco para doença arterial coronariana (DAC).

\section{Palavras-chave}

Doença arterial coronariana, angiografia coronariana, lipoproteína(a).

\section{Objective}

To determine serum levels of lipoprotein(a) and lipid profile of a group of individuals submitted to coronary angiography, with the aim of establishing the possible correlation between these parameters and the severity of coronary artery disease.

\section{Methods}

Serum levels of total cholesterol, $H D L_{c}, L D L_{c}$, triglycerides, lipoprotein(a), apolipoproteins $A-I$ and $B$ were measured in blood samples of 17 subjects with absence of atheromatosis in the coronary arteries (control), 12 subjects presenting mild/moderate atheromatosis and 28 subjects presenting severe atheromatosis.

\section{RESULTS}

No significant statistical differences were found between the means of the three groups for the parameters assessed, except for lipoprotein(a) serum levels which presented significant differences between the means of the control, mild/moderate atheromatosis and severe atheromatosis groups $(p<0.001)$.

\section{Conclusion}

The means obtained in the three groups for $L p(a)$ indicate a progressive increase in the serum levels of this parameter according to the severity of coronary atheromatosis. These findings suggest the need of additional studies in order to obtain enough evidence to support the introduction of routine assessment of $L p(a)$ levels in clinical laboratories in the monitoring of patients at risk for coronary artery disease (CAD).

\section{KEY WORDS}

Coronary artery disease, coronary angiography, lipoprotein(a).

Correspondência: Marinez de Oliveira Souza • Av. Antônio Carlos, 6627 - 31270-901 - Belo Horizonte, MG 
A doença arterial coronariana (DAC) apresenta origem multifatorial, compreendendo fatores de risco hereditários e adquiridos, que podem ser responsáveis diretos ou apenas estar associados à doença $a^{1,2}$. As alterações do metabolismo lipídico têm um papel relevante na evolução da aterosclerose ${ }^{3}$ e a avaliação laboratorial das lipoproteínas é de fundamental importância para o diagnóstico e tratamento da mesma4.

A lipoproteína(a) foi descrita por Berg em $1963^{5}$ como sendo uma variante genética da LDL. A Lp(a) apresenta uma composição lipídica similar à da LDL, e difere no conteúdo protéico, uma vez que apresenta a apolipoproteína(a) ou apo(a) ligada à apolipoproteína B através de pontes dissulfeto ${ }^{6-9}$. Os níveis plasmáticos de Lp(a) e a massa molecular da apo(a) são muito variáveis entre as pessoas, sendo determinados geneticamente ${ }^{10}$. A $L p(a)$ não tem qualquer função no transporte de lípides, portanto, sua ausência no plasma não acarreta transtornos metabólicos.

A apo(a) possui um alto grau de homologia com o plasminogênio, o precursor inativo da plasmina, proteína responsável pela quebra da fibrina produzida no processo de coagulação, devido ao variado número de repetições de seqüência de aminoácidos, homólogos à região do kring/e 4 do plasminogênio. Esta estrutura permite a ligação da $L p(a)$ à fibrina e às proteínas da superfície celular de células endoteliais e monócitos, bem como a inibição competitiva do ativador do plasminogênio tecidual (t-PA), diminuindo a geração de plasmina e a fibrinólise ${ }^{11}$. Estas características conferem à $L p(a)$ propriedades pró-aterogênicas ${ }^{12-14}$, estando os níveis elevados desta lipoproteína associados ao risco de DAC prematura, doença cerebrovascular e reestenose de lesões coronarianas. Alguns autores consideram a Lp(a) um fator de risco independente para aterosclerose coronariana e de artérias cerebrais ${ }^{10,14-16}$ em caucasianos, chineses, africanos e indianos ${ }^{9}$.

0 gene da apo(a) apresenta polimorfismo genético de tamanho, com mais de 30 alelos variando de 300 a $800 \mathrm{KDa}$, tendo sido sugerido que este polimorfismo possa contribuir para a elevação de $L p(a)$ em pacientes com $\mathrm{DAC}^{9,13}$. O real fator de risco parece estar associado à sub-população de $L p(a)$, sendo as isoformas de menor tamanho e maior afinidade pela fibrina, aquelas com maior associação com DAC ${ }^{11,13}$.

Um grande número de pacientes que desenvolvem DAC apresenta níveis lipídicos normais ou moderadamente elevados ${ }^{17}$, demonstrando que, apesar da enorme contribuição das dislipidemias para o desenvolvimento de DAC, outros fatores também são responsáveis pela progressão da aterosclerose. A Lp(a) está envolvida na patogênese e progressão da aterosclerose, através de diferentes mecanismos. Estudos epidemiológicos prospectivos e de meta-análise ${ }^{10,14,16,18}$, demonstraram a correlação positiva de $\mathrm{Lp}(\mathrm{a})$ e DAC. Frohlich e cols. ${ }^{14}$ sugeriram que a determinação de Lp(a) é importante ferramenta na avaliação de pacientes com DAC, sendo particularmente útil na predição de risco em mulheres. Entretanto, o desenvolvimento e a extensão da lesão da DAC estabelecida por angiografia e as correlações com os lípides plasmáticos e Lp(a), são controversas.

Acredita-se que um maior entendimento sobre as alterações lipídicas nas diversas fases de instalação da DAC possa constituir uma ferramenta em potencial para auxiliar a clínica no acompanhamento de pacientes com aterosclerose, fornecendo subsídios para a detecção precoce da gravidade da lesão.

Diante do contexto exposto acima, o presente estudo teve como objetivo investigar a existência de alterações nos níveis plasmáticos de $L p(a)$ e parâmetros do perfil lipídico em pacientes com diagnóstico de DAC estabelecido por angiografia e comparando-os com indivíduos angiograficamente normais, buscando correlacionar as possíveis alterações com a gravidade da lesão.

\section{Métodos}

Foram avaliados 57 indivíduos num período de três meses, com faixa etária de 46 a 68 anos, de ambos os sexos, selecionados no Departamento de Hemodinâmica do Hospital Socor de Belo Horizonte, após realização de angiografia coronariana. Os participantes deste estudo foram selecionados buscando uma homogeneidade em relação às variáveis sexo, idade, nível sócio-econômico e Índice de Massa Corporal (IMC). Com base nos resultados da angiografia coronariana, os componentes do presente trabalho foram divididos em três grupos: controle $(n=17)$, ateromatose leve/moderada $(n=12)$ e ateromatose grave $(n=28)$. A tabela 1 apresenta a caracterização dos grupos, com relação ao sexo, idade e IMC. 0 protocolo de estudo recebeu parecer favorável sob o ponto de vista ético e formal pelo Comitê de Ética em Pesquisa do Hospital Socor e pelo Comitê de Ética em Pesquisa da UFMG.

Aos indivíduos selecionados, foi feito o esclarecimento sobre os objetivos da pesquisa e, aqueles que estiveram de acordo, assinaram o Termo de Consentimento Livre e Esclarecido - TCLE. Uma ficha clínica de cada indivíduo, com identificação, dados demográficos, história familiar e resultado da angiografia coronariana foi preenchida pelos cardiologistas do Departamento de Hemodinâmica.

Foram excluídos do estudo indivíduos com história anterior (até 3 meses) de síndrome coronariana aguda (SCA); em uso de anticoagulantes orais, drogas hipolipemiantes ou estrogênios; portadores de doenças intercorrentes como distúrbios da coagulação, doenças renais, hepáticas e auto-imunes, diabetes mellitus, câncer e; indivíduos apresentando níveis de triglicérides acima de $400 \mathrm{mg} / \mathrm{dL}$.

A caracterização dos grupos quanto aos fatores de risco associados à DAC e presença de SCA anterior a três meses da entrevista é mostrada na tabela 1 , como número de indivíduos e porcentagem da presença de 
Tabela 1 - Caracterização dos grupos de estudo

\begin{tabular}{|c|c|c|c|}
\hline & Controle & Ateromatose leve/moderada & Ateromatose grave \\
\hline$n(M / F)$ & $17(8 / 9)$ & $12(8 / 4)$ & $28(16 / 12)$ \\
\hline Idade (anos) & $58,9 \pm 7,7$ & $61,0 \pm 11,6$ & $60,9 \pm 9,6$ \\
\hline $\mathrm{IMC}\left(\mathrm{Kg} / \mathrm{m}^{2}\right)$ & $24,9 \pm 4,6$ & $26,1 \pm 4,9$ & $24,3 \pm 3,2$ \\
\hline Tabagismo & $3(17,6 \%)$ & $3(25,0 \%)$ & $9(32,1 \%)$ \\
\hline Hipertensão arterial & $14(82,4 \%)$ & $9(75,0 \%)$ & $24(85,7 \%)$ \\
\hline Sedentarismo & $16(94,1 \%)$ & $9(75,0 \%)$ & $21(75,0 \%)$ \\
\hline História familiar & $7(41,2 \%)$ & $5(41,7 \%)$ & $12(42,9 \%)$ \\
\hline SCA anterior a 3 meses & $2(11,8 \%)$ & $4(33,3 \%)$ & $16(57,1 \%)^{\mathrm{a}}$ \\
\hline
\end{tabular}

determinada variável. A presença das variáveis tabagismo, sedentarismo e história familiar para DAC foi constatada com base nas recomendações das III Diretrizes Brasileiras sobre Dislipidemias e Prevenção de Aterosclerose 4 . Foram considerados hipertensos os participantes com diagnóstico prévio de hipertensão arterial que faziam uso regular de medicamentos hipotensores. Não houve diferença estatisticamente significativa para as variáveis tabagismo, hipertensão arterial, sedentarismo e história familiar entre os três grupos. Os participantes não apresentaram sobrepeso ou obesidade, sugerindo a ausência do componente metabólico nestes indivíduos. Para a variável SCA foram considerados os indivíduos que apresentaram infarto agudo do miocárdio ou angina instável. Houve diferença estatisticamente significativa entre os grupos controle e ateromatose grave $(p<0,01)$.

As amostras de sangue venoso foram obtidas com o paciente em jejum de 12 horas. Os mesmos foram orientados para não praticarem atividade física vigorosa e evitar a ingestão de etanol, nas 24 e 72 horas, respectivamente, que antecederam a coleta de sangue, na tentativa de obter amostras biológicas de pacientes em estado metabólico de equilíbrio. A coleta do sangue foi feita utilizando tubos do sistema Vacuette ${ }^{\circledR}$ (Geiner Bio-One), e tendo sido as amostras centrifugadas a $2.500 \mathrm{rpm}$ por 15 minutos para separação do soro, cujo material foi dividido em alíquotas e estocado a $-70^{\circ} \mathrm{C}$ por três meses.

O colesterol total e triglicérides foram determinados pelos métodos enzimáticos colorimétricos, Randox Cholesterol CHOD-PAP e Randox Triglycerides GPO-PAP, respectivamente. As determinações de $\mathrm{HDL}_{\mathrm{c}}$ e $\mathrm{LDL}_{\mathrm{c}}$ foram realizadas pelo método enzimático de eliminação da Randox HDL Cholesterol Direct e Randox LDL Cholesterol Direct, respectivamente. As dosagens de apo B e apo A-I foram realizadas pelos métodos turbidimétricos da Biotécnica, Apolipoproteína B e Apolipoproteína A-I, respectivamente. Os níveis plasmáticos de $L p(a)$ foram obtidos pelo método de turbidimetria, utilizando-se o conjunto diagnóstico In Vitro Lipoproteína(a). Todas as dosagens acima citadas foram realizadas no aparelho Cobas Mira Plus, utilizando-se soros-controle para verificar o desempenho dos ensaios. Foi utilizada a equação de Friedewald ${ }^{19} \mathrm{com}$ o intuito de se investigar o desempenho de dois processos diferentes para a quantificação de $L L_{C}$.

A angiografia coronariana foi realizada pela técnica de Judkins, os filmes foram examinados por três cardiologistas experientes e os laudos apresentados conforme critérios definidos pelo estreitamento da luz das artérias: até 30\% de estreitamento foi classificada como ateromatose leve; de 30 a $70 \%$ de estreitamento foi classificada como ateromatose moderada e acima de $70 \%$ de estreitamento foi classificada como ateromatose grave, em uma ou mais artérias afetadas.

Na análise estatística, o teste de análise de variância (ANOVA) foi utilizado para os parâmetros colesterol total, apo $\mathrm{B}, \mathrm{HDL}_{\mathrm{C}}, \mathrm{LDL}_{\mathrm{c}}$-direto e $\mathrm{LDL}_{\mathrm{c}}$-Friedewald, que apresentaram distribuição normal e homocedasticidade. $O$ teste de análise de variância Kruskal-Wallis foi utilizado para os parâmetros apo A-I e triglicérides, que não apresentaram distribuição normal e/ou homocedasticidade. O nível de significância adotado foi de 0,05. Especialmente para o parâmetro Lp(a), a análise de variância foi realizada pelo método Student-Newman-Keuls, após transformação logarítmica dos dados. Os programas Sigma Stat versão 1.0 e Prism versão 3.0 foram utilizados para realizar as análises e plotar o gráfico, respectivamente.

\section{Resultados}

Os resultados dos parâmetros bioquímicos são apresentados na tabela 2, como médias e respectivos desvios-padrão. Não houve diferenças estatisticamente significativas para os parâmetros colesterol total, $\mathrm{HDL}_{\mathrm{c}}$, $\mathrm{LDL}_{c}$, triglicérides, apo $\mathrm{A}-\mathrm{I}$ e apo $\mathrm{B}$. Para os níveis plasmáticos de $L p(a)$ foi observada diferença significativa entre o soro-controle e ateromatose leve/moderada $(p<0,0001)$, entre os grupos controle e ateromatose grave $(p<0,0001)$ e entre os grupos ateromatose leve/moderada e ateromatose grave $(p<0,001)$. A figura 1 apresenta a distribuição dos valores de $L p(a)$ nos grupos estudados.

A prevalência de níveis de $\mathrm{Lp}(\mathrm{a})$, maiores que $30 \mathrm{mg} / \mathrm{dL}$ foi de $0 \%, 50 \%$ e $67,9 \%$, respectivamente, para os grupos controle, ateromatose leve/moderada e ateromatose grave. 
Houve diferença significativa entre os grupos controle e ateromatose leve/moderada $(p<0,001)$ e entre os gruposcontrole e ateromatose grave $(p<0,0001)$.

\section{Discussão}

O desenvolvimento de testes laboratoriais capazes de identificar pacientes com maior risco de desenvolver DAC é preocupação de muitos pesquisadores e alvo de inúmeros estudos. A partir desse tipo de estudo, pode-se julgar a utilidade da determinação laboratorial de um dado parâmetro, no sentido de prever a doença, estabelecer sua extensão ou monitorar a eficácia do tratamento instituído.

Este estudo de coorte transversal avaliou uma população de risco intermediário a elevado, já que todos os indivíduos selecionados foram encaminhados a cateterismo para avaliação de dor torácica e apresentaram perfil epidemiológico e fatores de risco para DAC conforme mostrado na tabela 1 . Os três grupos avaliados apresentaram homogeneidade em relação à idade, sexo e IMC. Não foram observadas diferenças significativas entre as variáveis clínicas tabagismo, hipertensão arterial, história familiar e sedentarismo (tab. 1). Entretanto, observa-se que no grupo ateromatose grave houve uma maior incidência de indivíduos tabagistas e hipertensos em relação aos demais grupos. Entre os 22 indivíduos com história de SCA anterior a três meses, 16 (57,1\%) são portadores de ateromatose grave, apresentando diferença significativa em relação ao grupo controle (tab. 1).

$\mathrm{Na}$ avaliação do perfil lipídico, as médias dos três grupos não apresentaram diferenças estatisticamente significativas $(p<0,05)$ para os parâmetros colesterol total, $\mathrm{HDL}_{c}, \mathrm{LDL}_{c}$ (direto e estimado pela fórmula de Friedewald), triglicérides, bem como para as dosagens de apo A-I e apo B. Os valores das médias obtidas para colesterol total, $\mathrm{HDL}_{c}$, triglicérides, apo $\mathrm{A}-\mathrm{I}$ e apo $\mathrm{B}$ situam-se praticamente na faixa de valores desejáveis e limítrofes ${ }^{4}$, demonstrando que os indivíduos selecionados não apresentaram alterações significativas destes parâmetros (tab. 2). Resultado semelhante foi obtido em estudo com população indiana, em indivíduos com DAC estabelecida por angiografia ${ }^{20}$.
Com relação ao $\mathrm{LDL}_{\mathrm{c}}$, as médias obtidas neste estudo, para os grupos ateromatose leve/moderada e ateromatose grave, encontram-se acima dos níveis preconizados para indivíduos portadores de $\mathrm{DAC}^{4}$, demonstrando a presença de um importante fator causal e independente para aterosclerose e DAC nestes pacientes.

Com o intuito de se avaliar o desempenho de dois métodos diferentes para a determinação dos valores de $L L_{C}$, foi utilizada a equação de Friedewald, largamente utilizada na prática laboratorial para a estimativa dos valores de $\mathrm{LDL}_{\mathrm{C}}$, tendo sido observada uma correlação positiva da ordem de $96 \%$ com o $\mathrm{LDL}_{c}$ direto $(r=0,96 ; p<0,0001)$.

Para o parâmetro $L p(a)$, a diferença entre as médias foi estatisticamente significativa $(p<0,0001)$ entre os grupos-controle e ateromatose grave, entre os gruposcontrole e ateromatose leve/moderada $(p<0,0001)$ e entre os grupos ateromatose leve/moderada e ateromatose grave $(p<0,001)$ (tab. 2), confirmando a preocupação dos consensos americano, europeu e brasileiro em classificá-la como marcador de risco emergente e independente para DAC $^{1,2,4}$. Observa-se nos dados apresentados na tabela 2, que a média obtida para o grupo ateromatose leve/moderada apresenta-se quase três vezes maior que a média obtida para o grupo controle, e a média obtida para o grupo ateromatose grave apresenta-se cerca de cinco vezes maior que aquela apresentada pelo grupo controle e cerca de uma vez e meia maior que a média observada para o grupo ateromatose leve/moderada, sinalizando um aumento progressivo nos níveis plasmáticos de $L p(a)$ de acordo com a gravidade da aterosclerose coronariana. A análise dos resultados de $\mathrm{Lp}$ (a) mostrou, também, que no grupo-controle todos os 17 indivíduos apresentaram níveis de $L p(a)$ na faixa de referência do método (fig. 1). Nesta figura, observa-se que no grupo ateromatose leve/moderada $(n=12)$, apenas um indivíduo apresentou elevação significativa, com nível muito superior à média do grupo ateromatose grave, enquanto cinco outros indivíduos apresentaram elevações moderadas, porém abaixo desta média. Observa-se também que a média do grupo ateromatose leve/moderada situa-se no valor limítrofe da faixa de referência, já indicando uma tendência de elevação de Lp(a) neste grupo.

\section{Tabela 2 - Parâmetros bioquímicos em pacientes com coronárias isentas de ateromatose} e grupos com doença arterial coronariana (DAC)

\begin{tabular}{|c|c|c|c|}
\hline & Controle & Ateromatose leve/moderada & Ateromatose grave \\
\hline CT (mg/dL) & $209,1 \pm 53,5$ & $212,4 \pm 56,3$ & $203,1 \pm 42,7$ \\
\hline HDLc (mg/dL) & $50,0 \pm 9,2$ & $48,2 \pm 7,6$ & $48,1 \pm 7,8$ \\
\hline LDLc f (mg/dL) & $130,8 \pm 43,9$ & $131,6 \pm 48,8$ & $124,7 \pm 38,3$ \\
\hline LDLc d (mg/dL) & $139,4 \pm 45,7$ & $143,3 \pm 50,8$ & $135,8 \pm 37,0$ \\
\hline $\mathrm{TG}(\mathrm{mg} / \mathrm{dL})$ & $140,4 \pm 62,6$ & $162,9 \pm 69,4$ & $155,6 \pm 68,5$ \\
\hline Apo A-I (mg/dL) & $125,1 \pm 6,7$ & $124,2 \pm 5,9$ & $125,9 \pm 7,4$ \\
\hline Apo B (mg/dL) & $76,9 \pm 16,5$ & $81,3 \pm 17,4$ & $79,4 \pm 16,4$ \\
\hline Lp (a) (mg/dL) & $11,0 \pm 7,3$ & $29,4 \pm 25,6^{a}$ & $51,7 \pm 39,7$ a, b \\
\hline
\end{tabular}




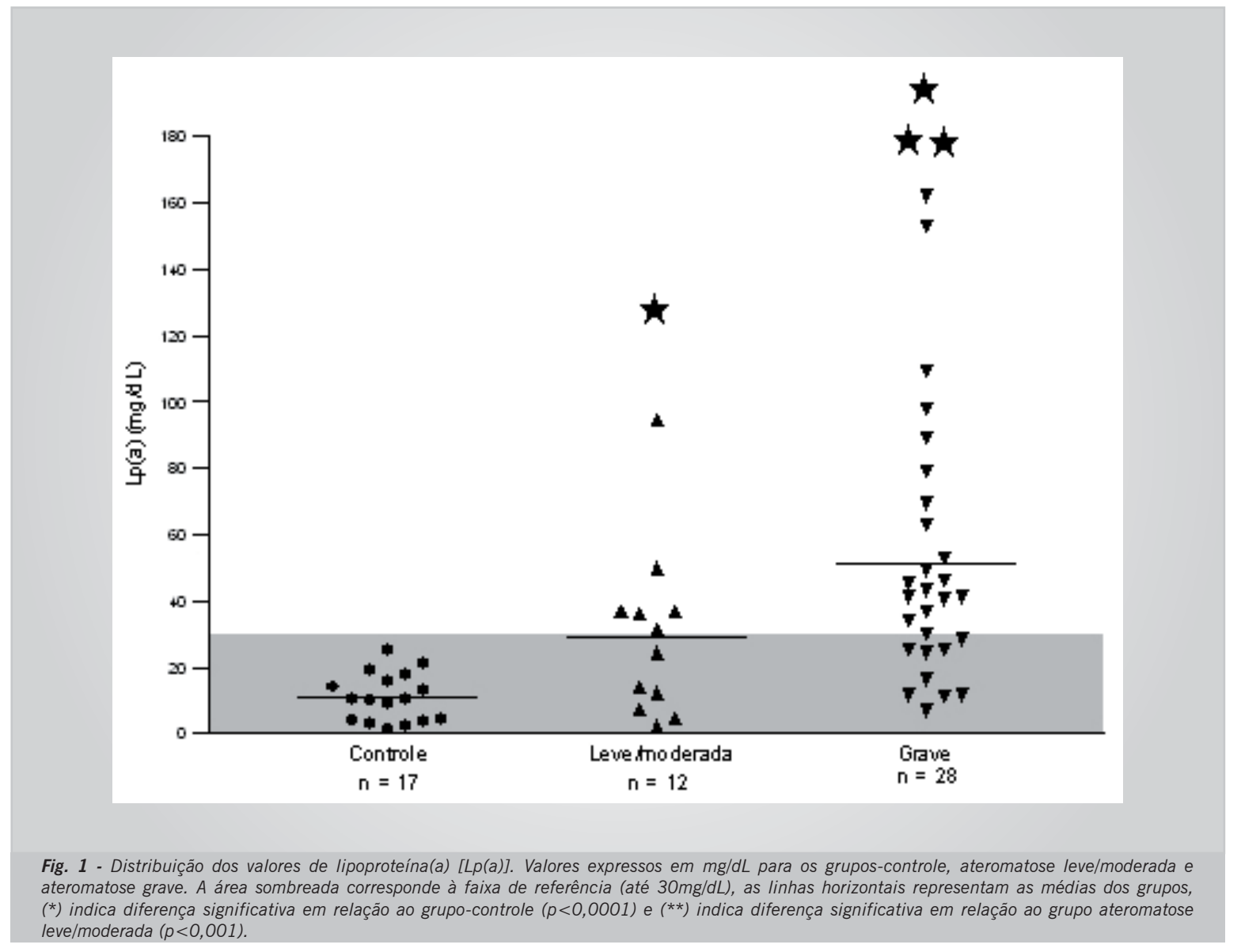

Correlação positiva entre Lp(a) e DAC estabelecida por angiografia foi demonstrada por Gupta e cols. ${ }^{20}$ na população indiana e também por Labeur e cols. ${ }^{8}$ na população belga, os quais observaram elevações dos valores de $L p(a)$ com o aumento da gravidade da DAC, nos casos mais graves com estenose significativa em mais de duas artérias coronárias. Na população brasileira, Maranhão e cols. ${ }^{21}$ demonstraram a associação entre níveis plasmáticos elevados de Lp(a) e a extensão da DAC em caucasianos brasileiros, submetidos à cinecoronariografia.

Não foram observadas correlações de Lp(a) com outras variáveis do perfil lipídico nos indivíduos estudados, tendo sido descrito resultado semelhante por Genest e cols. ${ }^{10}$. Entretanto, Labeur e cols. ${ }^{8}$ descreveram correlações significativas entre $L p(a)$ e $L D L_{c}$, estimadas pela fórmula de Friedewald, bem como a correlação inversa de $L p(a)$ e triglicérides.

Houve diferenças significativas quanto à prevalência de níveis elevados de $L p(a)$ entre os grupos controle e ateromatose leve/moderada ( $p<0,001)$ e entre os gruposcontrole e ateromatose grave $(p<0,0001)$. A elevação de $L p(a)$ no grupo ateromatose grave foi altamente prevalente $(67,9 \%)$. A avaliação da prevalência de níveis elevados de $L p(a)$ independente do grau de estenose (ateromatose leve/moderada e grave, $n=40$ ), demonstra um elevado percentual (62,5\%). Foi observada diferença significativa entre este grupo com relação ao grupo- controle $(p<0,01)$.

A análise da tabela 1 e figura 1 permite inferir sobre a existência de uma relação não linear entre $L p(a)$ e risco de desenvolvimento de DAC na população avaliada, considerando a falta de significância com relação aos fatores de risco nos três grupos estudados. Portanto, a Lp(a) não se comportou no estudo como um fator de risco mas sim como marcador ou determinante de risco cardiovascular, uma vez que não foi possível estabelecer uma relação de causa e efeito.

Alguns estudos demonstram que o valor preditivo da Lp(a) para a gravidade e extensão da DAC seria maior em mulheres ${ }^{14,21}$; entretanto, o presente estudo não evidenciou diferença significativa entre a prevalência de níveis elevados de Lp(a) entre homens e mulheres na população estudada. Por outro lado, alguns estudos não demonstraram correlação entre níveis plasmáticos de $\mathrm{Lp}(\mathrm{a})$ e $\mathrm{DAC}^{22}$ e outros atribuem o real valor preditivo à sub-população de Lp(a) com alta afinidade por fibrina ${ }^{11}$. De acordo com este conceito, alguns fenótipos de Lp(a) não estariam associados à aterotrombose e nem todos os indivíduos com elevação de Lp(a) apresentariam risco aumentado de DAC. Na população brasileira, que apresenta uma heterogeneidade de etnias, são raros os estudos deste tipo. 0 grupo estudado neste trabalho $(n=57)$ constou de $28,1 \%$ de caucasianos, $22,8 \%$ de negros e $49,1 \%$ de mestiços (mulato, pardo ou outros 
mestiços), não apresentando diferenças estatisticamente significativas entre os três grupos para as diferentes etnias. Maranhão e cols. ${ }^{21}$ demonstraram valores significativamente mais elevados em indivíduos brasileiros da raça negra, quando comparados com os caucasianos. Entretanto, os valores mais altos na população de raça negra não foram associados com a presença ou extensão da DAC na população avaliada. A análise multivariada deste estudo demonstrou que a presença de níveis elevados de Lp(a) contribuíram para o desenvolvimento de DAC em caucasianos brasileiros.

Em conclusão, os dados deste estudo confirmam a utilidade da $L p(a)$ para predizer a gravidade da aterosclerose coronariana, sugerindo que os níveis de Lp(a) devem ser determinados em pacientes com DAC, especialmente em normolipêmicos, uma vez que a Lp(a) comportou-se como um marcador de predição de gravidade da aterosclerose coronariana, independente de tabagismo, hipertensão arterial, sedentarismo, história familiar e perfil lipídico. À luz do presente conhecimento sobre o assunto, diante das notáveis controvérsias, e com base nos dados ora apresentados, espera-se que este trabalho possa suscitar ânimos para a realização de outros estudos envolvendo a medida da $L p(a)$ em indivíduos normais e com graus crescentes de obstrução coronariana, confirmada angiograficamente, em nossa população. Apesar do conhecimento da existência de diversas isoformas de $\mathrm{Lp}(\mathrm{a})^{12}$ que podem dificultar o desempenho do sistema fibrinolítico, provocando maior ou menor inibição deste, considera-se altamente relevante o fato de que todos os componentes do grupo com ateromatose grave apresentaram níveis plasmáticos de $L p(a)$ muito elevados, quando comparados aos níveis apresentados pelos indivíduos normais, todos comprovados angiograficamente. Mesmo que as diversas variantes de $L p(a)$ possam afetar com intensidades diferentes a eficiência do sistema fibrinolítico, deve ser motivo de preocupação e de constante investigação a relação entre $L p(a)$ e doença coronariana, notadamente em indivíduos normocolesterolêmicos. Uma análise dos dados encontrados permite inferir sobre a utilidade de se introduzir a determinação de Lp(a) na avaliação de indivíduos que apresentam risco aumentado para DAC, mas não na estratificação de risco da população em geral. A assertiva acima é também sustentada pela contínua e crescente associação entre níveis elevados de $L p(a)$ e risco para doença cardiovascular ${ }^{6,14-16,23-25}$, bem como pela possibilidade de adoção de medidas terapêuticas e de controle 26,27. $^{2}$.

Embora os valores da $L p(a)$ ainda não sejam utilizados para determinar a gravidade da DAC, estes podem ter um papel relevante e adicional na avaliação do desenvolvimento e especialmente, na evolução da DAC. Esta linha de pesquisa representa um desafio para várias áreas de estudo do envolvimento da $L p(a)$ e outros fatores na patogênese e na progressão da aterosclerose.

\section{Agradecimentos}

Ao Prof. Dr. Ivan Barbosa Machado Sampaio, pelo auxílio na análise estatística. À Fundação de Amparo à Pesquisa de Minas Gerais (FAPEMIG), à Coordenação de Aperfeiçoamento de Pessoal de Nível Superior (CAPES), ao Conselho Nacional de Desenvolvimento Científico e Tecnológico (CNPq), ao LABORATEL Laboratório de Análises Clínicas, à IN VITRO Diagnósticos e à BIOTÉCNICA Biotecnologia Avançada, pelo apoio recebido.

\section{Potencial Conflito de Interesses}

Declaro não haver conflitos de interesses pertinentes.

\section{REFERÊNCIAS}

1. American Heart Association (AHA). NCEP Report: Implications of Recent Clinical Trials for the National Cholesterol Education Program Adult Treatment Panel III Guidelines. Circulation 2004;110:227-39.

2. European Society of Cardiology (ESC). European guidelines on cardiovascular disease prevention in clinical practice: Third Joint Task Force of European and Other Societies on Cardiovascular Disease Prevention in Clinical Practice. Eur J Card Prev Reh 2003;10(Supl I) : S1-78.

3. Sharrett AR, Ballantyne CM, Coady SA, et al. Coronary heart disease prediction from lipoprotein cholesterol levels, triglycerides, lipoprotein(a), apolipoproteins A-I and B, and HDL density subfractions. Circulation 2001;104:1108-13.

4. Sociedade Brasileira de Cardiologia (SBC). III Diretrizes Brasileiras Sobre Dislipidemias e Diretrizes de Prevenção da Aterosclerose. Arq Bras Cardiol 2001;77(Supl III):1-48.

5. Berg K. A new serum type system in man-the Lp system. Acta Pathol Scand 1963;59:382-86.

6. Koschinsky ML. Lipoprotein(a) and the link between atherosclerosis and trombosis. Can J Cardiol 2004;20(Supl B):37B-43B.

7. Hajjar KA, Nachman RL. The role of lipoprotein(a) in atherogenesis and thrombosis. Ann Rev Med 1996;47:423-42.

8. Lauber C, De Bacquer D, De Backer G, et al. Plasma lipoprotein(a) values and severity of coronary artery disease in a large population of patients undergoing coronary angiography. Clin Chem 1992;38:2261-6.

9. Pati U, Pati N. Lipoprotein(a), atherosclerosis, and apolipoprotein(a) gene polymorphism. Mol Gen Met 2000;71:87-92.

10. Genest J, Jenner JL, McNamara JR, etal. Prevalence of lipoprotein(a) [Lp(a)] excess in coronary artery disease. Am J Cardiol 1991;67:1039-45.

11. Anglés-Cano E, Penã-Díaz A, Loyau S. Inhibition of fibrinolysis by lipoprotein(a). Ann N Y Acad Sci 2001;936:261-75.

12. Hancock MA, Boffa MB, Marcovina SM, et al. Inhibition of plasminogen activation by lipoprotein(a): critical domains in apolipoprotein(a) and mechanism of inhibition on fibrin and degraded fibrin surfaces. J Biol Chem 2003;278:23260-9.

13. Peña-Díaz A, Izaguirre-Avila R, Anglés-Cano E. Lipoprotein Lp(a) and atherothrombotic disease. Arch Med Res 2000;31:353-59.

14. Frohlich J, Dobíasova M, Adler L, et al. Gender differences in plasma levels of lipoprotein(a) in patients with angiographically proven coronary artery disease. Physiol Res 2004;53:481-6.

15. Fujino A, Watanabe T, Kunii H, et al. Lipoprotein(a) is a potential coronary risk factor. Jpn Circ J 2000;64:51-56. 
16. Luc G, Bard JM, Arveiler D, et al. Lipoprotein(a) as a predictor of coronary heart disease: the PRIME study. Atherosclerosis 2002;163:377-84.

17. Cantin B, Després JP, Lamarche B, et al. Association of fibrinogen and lipoprotein(a) as a coronary heart disease risk factor in men (The Quebec Cardiovascular Study). Am J Cardiol 2002;89:662-66.

18. Danesh J, Collins R, Peto R. Lipoprotein(a) and coronary heart disease: meta-analysis of prospective studies. Circulation 2000; 102:1082-5.

19. Friedewald WT, Levy RI, Fredickison DS. Estimation of the concentration of low density lipoprotein cholesterol in plasma, without the use of preparative ultracentrifugue. Clin Chem 1972; 18:499-52.

20. Gupta R, Vasisht S, Bahl VK, et al. Correlation of lipoprotein(a) to angiographically defined coronary artery disease in Indians. Int J Cardiol 1996; 57:265-70.

21. Maranhão RC, Vinagre CG, Arie S, et al. Lipoprotein(a) in subjects with or without coronary artery disease: relation to clinical history and risk factors. Braz J Med Biol Res 1995;28:439-46.
22. Cantin B, Gagnon F, Moorjani S, et al. Is lipoprotein(a) an independent risk factor for ischemic heart disease in men? The Quebec Cardiovascular Study. J Am Coll Cardiol 1998; 31:519-25.

23. Cremer P, Nagel D, Labrot NB, et al. Lipoprotein(a) as a predictor of acute myocardial infarction in comparison to fibrinogen, LDL cholesterol and other risk factors: results from the prospective Gottingen risk incidence and prevalence study. Eur J Clin Invest 1994; 24: 444-53.

24. Schaefer EJ, Lamon-Fava S, Jenner JL, et al. Lipoprotein(a) levels and risk of coronary heart disease in men: the lipidic research clinics coronary primary prevention trial. JAMA 1994; 271: 999-1003.

25. Marcovina SM, Koschinsky ML. A critical evaluation of the role of Lp(a) in cardiovascular disease: can Lp(a) be useful in risk assessment? Semin Vasc Med 2002; 2:335-44.

26. Sposito AC, Mansur AP, Maranhão RC, et al. Etofibrate but not controlledrelease niacin decreases $L D L$ cholesterol and lipoprotein(a) in type IIb dyslipidemic subjects. Braz J Med Biol Res 2001; 34: 177-82.

27. Mikhailidis DP, Ganotakis ES, Spyropoulos KA, et al. Prothrombotic and lipoprotein variables in patients attending a cardiovascular risk management clinic: response to ciprofibrate of lifestyle advice. Int Angiol 1998; 17: 225-33. 